\title{
COMPARATIVE PERFORMANCE OF IN-HOUSE AND COMMERCIALLY AVAILABLE ELISA KITS FOR THE DETECTION OF ANTIBODY AGAINST NEWCASTLE DISEASE VIRUS VACCINE
}

\author{
M. M. I. Chowdhury, M. T. Islam ${ }^{1}$, A. Aktar, M. K. J. Bhuiyan ${ }^{2}$, M. M. Kamal, \\ M. A. Haque, M. J. Ara, M. M. Rahman and M. A. Islam* \\ Department of Microbiology and Hygiene, Bangladesh Agricultural University, \\ Mymensingh-2202, Bangladesh
}

\begin{abstract}
An In-house Indirect enzyme-linked immunosorbent assay (ELISA) was developed for the detection of serum antibody titre against Newcastle disease virus (NDV) and was compared its sensitivity and specificity with the commercially available NDV antibody detection ELISA kit (Biocheck ${ }^{\circledR}$, USA). The reference NDV was purified by centrifugation, ultracentrifugation and by sucrose density gradient ultracentrifugation. This purified NDV was used for coating of 96-well flat bottomed microtitre plate and to raise hyperimmune sera (known) in Fayoumi chickens. In the standardization test, the antigen dilution of $10^{-6}$ and the serum dilution of $10^{-3}$ were considered to be optimum for the present ELISA system. The correlation regression analysis was performed to construct a standard curve equation where a good positive correlation was observed $(\mathrm{r}=0.912, \mathrm{n}=8, \mathrm{P}<0.01)$. The equation was used to convert corrected absorbance readings of the single working dilution $(1: 1000)$ directly into predicted ELISA antibody activity titres. In the sensitivity and specificity test, the serum dilution of $10^{-5}$ appeared to be the highest dilution which had the maximum lowest capacity to bind with the coated antigen of the present ELISA kit and only antiNDV serum was found to bind with the coated antigen instead of serum of IBDV in the plate which revealed the high specificity of the developed In-house Indirect ELISA kit. In a comparative study with the 80 chicken sera samples, a significant positive correlation $(r=0.901, n=80, P<0.01)$ was found between In-house Indirect ELISA and commercial ELISA kit (Biocheck ${ }^{\circledR}$, USA).
\end{abstract}

Key Words: Standardization, Sensitivity, Specificity, ELISA kit, Antibody, Newcastle disease virus

\section{INTRODUCTION}

Few years ago, enzyme immunoassays were not used widely as routine clinical tests, rather used as research tools with some 'potential' diagnostic significance. However, due to rapid progress in the field of diagnosis the test has been made easy and economically

\footnotetext{
1 Department of Medicine, 2Department of Agricultural Statistics, Bangladesh Agricultural University, Mymensingh-2202, Bangladesh

Corresponding author: E-mail: alim_bau@yahoo.co.in; Cell: 01714-325562
} 
feasible with respect to the development and validation of these assays, and in many of test instances these versatile tests have been recognized as the choice for the detection of antibody in various species of animal against different organisms.

In many under developed and developed countries the ND appears commonly as enzootic and epizootic forms among the layer and broiler poultry in the breeder and commercial farms every year. Vaccination of chicken with either killed or live NDV vaccines may induce primarily active immunity (Alexander, 1991 and 1997). After vaccination immunity of a flock can be measured by several serological tests (Tizzard, 1982 and Alexander, 1991). The serum antibody level produced against NDV can be determined by haemagglutination inhibition (HI) test, virus neutralization (VN) test, enzyme-linked immunosorbent assay (ELISA), fluorescent antibody technique (FAT), plaque reduction neutralization test, agar gel immunodiffusion test (AGIDT) etc.

Though $\mathrm{HI}$ is been used as the standard test but recently the HI test has been replaced by enzyme-linked-immunosorbent assay (ELISA) (Brown et al., 1990 and Alexander, 1991) and considered as the more authentic, reliable and top categories of serological method than the HI test because of its high sensitivity and specificity. The farmers can easily determine the quality of the vaccine by using the In-house Indirect ELISA kit and can easily select the highly effective vaccine before vaccination their flock and easily measuring the antibody level of a flock of bird before and after vaccination. The In-house indirect ELISA system is a laboratory based and very easy to perform serological test method. Therefore, the goal of this research work were to evaluate the sensitivity and specificity of the newly developed In-house Indirect ELISA kit for measuring the level of serum antibody in a flock of birds before and after vaccination and comparison of the sensitivity and specificity of the newly developed In-house Indirect ELISA kit with that of the commercially available Newcastle disease virus ELISA kit.

\section{MATERIALS AND METHODS}

\section{Preparation of antigen for ELISA}

For the concentration and purification of NDV, infective allantoic fluid (AF) was used as a soul source. Separation of viral particles was done from non-viral elements present in the $\mathrm{AF}$ by centrifugation of the AF at 5,000 rpm for 30 minutes. Collection of the clear AF from the previous step for the concentration of virus and the pellet of the step was discarded from the fluid and the fluid was treated with PEG-6000 (6.6\%) and $\mathrm{NaCl}(2.2 \%)$. PEG-6000 $(6.6 \%)$ and $\mathrm{NaCl}(2.2 \%)$ treated clear $\mathrm{AF}$ was allowed to stir for overnight for complete binding of the virus with the PEG-6000 (6.6\%) keeping at $4^{\circ} \mathrm{C}$. Concentration of PEG treated virus was performed by high speed centrifugation at 12,000 rpm for 1 hour maintaining $4^{\circ} \mathrm{C}$. Supernatant of the bucket was discarded and the pellet was resuspended with 1X dPBS to get crude concentrated viral particles of NDV. Preparation of gradient column was done using different concentration (50\%-15\%) of sucrose solution in sterile dPBS. To get purified viral particles, the PEG treated crude concentrated virus was overlayed on the top layer of the column of sucrose (15\%) and was centrifuged at 16,000 
rpm for 2 hours maintaining $4^{\circ} \mathrm{C}$. Collection of $1 \mathrm{ml}$ of sucrose from each gradient solution was performed in separate glass tube to get various concentrations of purified ND viruses. More than one glass tubes contained similar concentration of virus and sucrose mixture were pooled and further concentration was done by centrifugation at 16,000 rpm for 2 hours. The supernatant was discarded and resuspension of the pellet was done to reconfirm the presence of NDV by slide HA test and the purified and concentrated virus was collected in cryo-vials for further used. The purified ND viruses were stored at $-80^{\circ} \mathrm{C}$ until used for coating of microtitre plate to develop an In-house Indirect ELISA kit.

\section{Coating of the 96-well plate with purified ND virus}

The NDV antigen was diluted appropriately in coating buffer ( $\mathrm{pH} 9.6)$ and diluted antigen $(100 \mu \mathrm{g})$ was dispensed to each well of an ELISA plate. The coated plate was incubated at $37^{\circ} \mathrm{C}$ for $1 \mathrm{~h}$, followed by overnight at $4^{\circ} \mathrm{C}$. The excess antigen was discarded and the plate was washed 5 times with approximately $200 \mu$ of washing buffer solution for each well. Uncoated sites of the wells were blocked with blockace solution to each well. After blocking the plate was incubated at $37^{\circ} \mathrm{C}$ for $1 \mathrm{~h}$. Blockace solution was discarded and the plate was washed 5 times with approximately $200 \mu \mathrm{l}$ of washing buffer solution for each well. Then the antigen coated microtitre plate was stored at $4^{\circ} \mathrm{C}$ until use.

\section{Antiserum preparation}

The inactivated NDV and IBDV viruses were then used for the preparation of the adjuvanted vaccine. Equal volume $(250 \mu \mathrm{l})$ of Freund's complete adjuvant was added with the formaldehyde inactivated viruses for the preparation of adjuvanted vaccine. Inactivated virus and adjuvant were mixed properly by using double nozzle needle and tuberculin syringe. Four chickens of 6 weeks of age were divided into two groups (Group " $A$ " and " $B$ "). The inactivated NDV vaccine was inoculated into two chickens in group " $\mathrm{A}$ " and the IBDV vaccine was inoculated into another two chickens in group " $\mathrm{B}$ " for three times at day 0,14 and 28 . About $5 \mathrm{ml}$ of blood was collected aseptically (using $5 \mathrm{ml}$ syringe without anticoagulant) from the wing vein of each experimental chickens of group " $\mathrm{A}$ " and group " $\mathrm{B}$ " at 14 days after $1^{\text {st }}, 2^{\text {nd }}$ and last vaccination. After collection of blood, the blood was allowed to clot in the syringe and then the serum was separated from the clot by traction and placing at $37^{\circ} \mathrm{C}$ for 1 hour. The straw colored fluid was then collected after incubation and centrifuged at 1,500 rpm for 10 minutes to obtain more clear serum. Micro-plate HI test was conducted to determine the HI titre of hyperimmune serum samples collected before and after vaccination with the purified inactivated NDV respectively in this study. The serum was finally collected in the screw-capped vial and preserved at $-20^{\circ} \mathrm{C}$ until use.

\section{Standardization of the newly developed In-house Indirect ELISA kit}

The known NDV serum and IBDV serum raised in chickens were used as undiluted and diluted at several concentration such as $10^{\circ}$ (undiluted), $10^{-1}, 10^{-2}, 10^{-3}, 10^{-4}, 10^{-5}, 10^{-6}, 10^{-7}, 10^{-8}$ and $10^{-9}$ with sample diluent to standardize the newly developed In-house Indirect ELISA kit. 


\section{Prediction of antibody titre}

A standard curve equation was derived to predict the antibody titre of a serum from the absorbance value obtained at a single dilution $(1: 1000)$. For this, eight serum samples having a range of absorbance values were selected. The end-point ELISA titre of all these eight sera was determined by serial 10-fold dilution. The end-point titres of these eight sera and the corresponding absorbance values at $1: 1000$ dilutions were subjected to correlation-regression analysis to derive a standard curve equation. This equation was used for predicting titre of a serum from the absorbance value at $1: 1000$.

\section{Sensitivity test of the newly developed In-house Indirect ELISA kit}

The hyperimmune NDV serum raised in chickens was diluted at $1: 1000$ dilutions. The sensitivity test of newly developed kit was performed by using different dilution of diluted hyperimmune NDV serum in which the antigen coated plate was abled to capture the maximum lowest concentration of serum. The known diluted hyperimmune serum was diluted at several concentrations such as $10^{-3}, 10^{-4}, 10^{-5}, 10^{-6}, 10^{-7}, 10^{-8}, 10^{-9}$ and $10^{-10}$ with sample diluent to evaluate the sensitivity test of the newly developed In-house indirect ELISA kit respectively.

\section{Specificity test of the newly developed In-house Indirect ELISA kit}

The specificity test of developed In-house Indirect ELISA kit was performed by using different samples like NDV, IBDV serum at $10^{-3}$ dilution to show the specificity of the newly developed In-house Indirect ELISA kit designed for specific binding of NDV serum only.

Comparison to the sensitivity and specificity of the newly developed In-house Indirect NDV ELISA kit with the commercially available Newcastle disease virus antibody detection ELISA kit

The performance of the ELISA system developed in the laboratory (In-house Indirect ELISA kit) was compared with that of a commercial ELISA kit. The commercial ELISA kit (BioChek, NDV antibody ELISA kit) was performed according to the manufacturer's instruction using pre-coated plates and pre-diluted, ready to use reagents and buffer. In case of Biochek NDV antibody ELISA kit, the titre was predicted from the absorbance value of 1:500 dilution of a serum using the formula supplied with the kit.

Both the newly developed In-house Indirect ELISA and commercial (Biochek, NDV antibody detection kit) ELISA were applied on 80 sera obtained from the vaccinated and recovered flocks of birds from commercial broiler and layer farms of Gazipur and BAU poultry farms. ELISA titre was predicted for each serum using both the systems.

\section{RESULTS AND DISCUSSION}

An indirect ELISA method was developed for the detection of antibody level of chicken raised against NDV in vaccinated and recovered flocks of birds. A computational method 
was also developed for predicting antibody titre from the absorbance value at a single dilution (1:1000) of the serum. The ELISA titre of 80 serum sample of chicken predicted by the developed ELISA system (In-house Indirect ELISA) correlated with that of commercial ELISA kit (Biochek NDV antibody detection kit).

Highly purified NDV antigen, prepared by ultracentrifugation, is generally used to develop ELISA kit (Folitse et al., 1998). Sucrose density gradient ultracentrifugation of NDV antigen was also used to develop ELISA kit (Williams et al., 1997). In the present study, the ELISA antigen was purified and concentrated from NDV infected allantoic fluid by high speed centrifugation followed by sucrose density gradient centrifugation method. This procedure resulted in a very good recovery of the antigen, as this was used at as high as $10^{-6}$. Nevertheless, a good discrimination between the positive (NDV serum) and negative serum was achieved from this study.

Micro-plate HI test was conducted to determine the HI titre of hyperimmune serum samples collected from each of the two chickens before and after vaccination with the purified inactivated NDV respectively in this study. Sera samples of vaccinated and nonvaccinated control groups of chicken were tested with the serum samples collected at day 14 and day 28 of $1^{\text {st }}$ and $2^{\text {nd }}$ vaccination and also with the hyperimmune serum collected at day $7^{\text {th }}$ of last vaccination by micro-plate HI test. The HI titre of hyperimmune serum of chickens vaccinated with third doses of purified inactivated NDV revealed that the HI titre of their serum was higher compare to the serum of other two vaccination groups 1536 $\pm 0,192 \pm 0$ and $384 \pm 0$ respectively in this study. This hyperimmune serum was used for standardization of newly developed In-house Indirect ELISA kit.

Ten fold serial dilution of the virus antigen starting from $10^{-5}$ up to $10^{-8}$ were used for coating the plate in this study. Then dilutions of the positive (NDV serum) and negative serum pools raised in chickens starting from $10^{\circ}$ up to $10^{-9}$ were used. In this trial, the best discrimination between the absorbance values of the positive (NDV serum) and negative serum pools were observed at the antigen dilution of $10^{-6}$ and $10^{-7}$. The differences were best observed at the serum dilutions of $10^{-2}$ and $10^{-3}$. Although the absorbance value of the positive (NDV serum) serum pool was almost double of that of the negative serum pool at $10^{-2}$ serum dilution, the absorbance value of 0.984 for the negative serum at this dilution indicated a high background reaction. So, the serum dilution of $10^{-3}$ was considered to be optimum and a dilution of 1:1000000 appeared to be the suitable concentration of antigen for the present ELISA system shown in Tables 1 and 2. Snyder et al. (1982) used $1: 1200$ dilution of antigen for coating the plate.

Eight serum samples were selected randomly on the basis of the absorbance values ranging from 0.266 to 1.256 at $10^{-3}$ dilution and titrated in ten-fold serial dilutions. The positive-negative threshold (PNT) baseline was drawn as the mean plus thrice the 
standard deviation of the absorbance values of six control sera, measured separately at $1: 10^{2}$ and $1: 10^{3}$ dilutions (Table 3). To determine the end-point titre, serial absorbance of each serum was plotted against $\log _{10}$ corresponding serum dilution. The point at which absorbance curve intersected the PNT baseline was considered as the end-point titre (Fig. 1).

Table 1. Standardization of optimal dilutions of the virus antigen and serum (results with the positive serum pool)

\begin{tabular}{l|c|c|c|c|c|c|c|c|c|c}
\hline \multirow{2}{*}{$\begin{array}{c}\text { Virus } \\
\text { dilution }\end{array}$} & \multicolumn{10}{c}{ OD value of Indirect ELISA } \\
\cline { 2 - 11 } & $10^{0}$ & $10^{-1}$ & $10^{-2}$ & $10^{-3}$ & $10^{-4}$ & $10^{-5}$ & $10^{-6}$ & $10^{-7}$ & $10^{-8}$ & $10^{-9}$ \\
\hline $10^{-5}$ & 2.255 & 2.205 & 2.005 & 1.305 & 0.805 & 0.756 & 0.616 & 0.748 & 0.656 & 0.612 \\
$10^{-5}$ & 2.194 & 2.158 & 2.116 & 1.285 & 0.775 & 0.711 & 0.685 & 0.618 & 0.714 & 0.678 \\
$10^{-6}$ & 2.174 & 1.953 & 1.655 & $\mathbf{0 . 8 9 5}$ & 0.178 & 0.168 & 0.156 & 0.143 & 0.178 & 0.163 \\
$10^{-6}$ & 2.150 & 1.876 & 1.592 & $\mathbf{0 . 7 5 5}$ & 0.165 & 0.175 & 0.163 & 0.123 & 0.136 & 0.131 \\
$10^{-7}$ & 1.789 & 1.715 & 1.475 & $\mathbf{0 . 7 4 1}$ & 0.155 & 0.134 & 0.128 & 0.154 & 0.129 & 0.125 \\
$10^{-7}$ & 1.749 & 1.696 & 1.405 & $\mathbf{0 . 7 9 3}$ & 0.146 & 0.144 & 0.145 & 0.135 & 0.156 & 0.129 \\
$10^{-8}$ & 1.865 & 1.719 & 1.325 & 0.385 & 0.166 & 0.157 & 0.198 & 0.139 & 0.141 & 0.131 \\
$10^{-8}$ & 1.814 & 1.678 & 1.295 & 0.365 & 0.159 & 0.143 & 0.171 & 0.145 & 0.155 & 0.124 \\
\hline
\end{tabular}

Table 2. Standardization of optimal dilutions of the virus antigen and serum dilution (results with the negative serum pool)

\begin{tabular}{l|c|c|c|c|c|c|c|c|c|c}
\hline \multirow{2}{*}{$\begin{array}{c}\text { Virus } \\
\text { dilution }\end{array}$} & \multicolumn{10}{c}{ OD value of Indirect ELISA } \\
\cline { 2 - 11 } & $10^{0}$ & $10^{-1}$ & $10^{-2}$ & $10^{-3}$ & $10^{-4}$ & $10^{-5}$ & $10^{-6}$ & $10^{-7}$ & $10^{-8}$ & $10^{-9}$ \\
\hline $10^{-5}$ & 3.125 & 2.856 & 1.371 & 0.984 & 0.729 & 0.696 & 0.625 & 0.745 & 0.718 & 0.654 \\
$10^{-5}$ & 3.331 & 2.759 & 1.213 & 0.816 & 0.681 & 0.611 & 0.714 & 0.655 & 0.711 & 0.756 \\
$10^{-6}$ & 3.183 & 2.655 & 0.898 & $\mathbf{0 . 4 8 3}$ & 0.179 & 0.164 & 0.149 & 0.141 & 0.166 & 0.156 \\
$10^{-6}$ & 3.151 & 2.615 & 0.877 & $\mathbf{0 . 3 9 2}$ & 0.172 & 0.165 & 0.161 & 0.134 & 0.128 & 0.133 \\
$10^{-7}$ & 2.718 & 2.156 & 0.919 & $\mathbf{0 . 3 8 3}$ & 0.144 & 0.124 & 0.131 & 0.144 & 0.133 & 0.124 \\
$10^{-7}$ & 2.598 & 1.956 & 0.802 & $\mathbf{0 . 3 8 9}$ & 0.125 & 0.137 & 0.136 & 0.129 & 0.151 & 0.121 \\
$10^{-8}$ & 2.526 & 1.896 & 0.756 & 0.181 & 0.153 & 0.147 & 0.185 & 0.133 & 0.139 & 0.139 \\
$10^{-8}$ & 2.424 & 1.786 & 0.685 & 0.147 & 0.162 & 0.152 & 0.165 & 0.141 & 0.145 & 0.21 \\
\hline
\end{tabular}

Table 3. Mean and standard deviation of the absorbance value of six control sera at $1: 10^{3}$ dilution

\begin{tabular}{|c|c|c|c|c|c|c|c|c|c|}
\hline \multirow[t]{2}{*}{ Serum dilution } & \multicolumn{6}{|c|}{ OD value } & \multirow[t]{2}{*}{ Mean } & \multirow{2}{*}{$\begin{array}{l}\text { Standard } \\
\text { deviation }\end{array}$} & \multirow[t]{2}{*}{ Mean $\pm 3 S D$} \\
\hline & 1 & 2 & 3 & 4 & 5 & 6 & & & \\
\hline $10^{-2}$ & 0.356 & 0.267 & 0.232 & 0.258 & 0.278 & 0.343 & 0.288 & 0.05 & 0.44 \\
\hline $10^{-3}$ & 0.275 & 0.198 & 0.169 & 0.174 & 0.222 & 0.263 & 0.217 & 0.04 & 0.35 \\
\hline
\end{tabular}




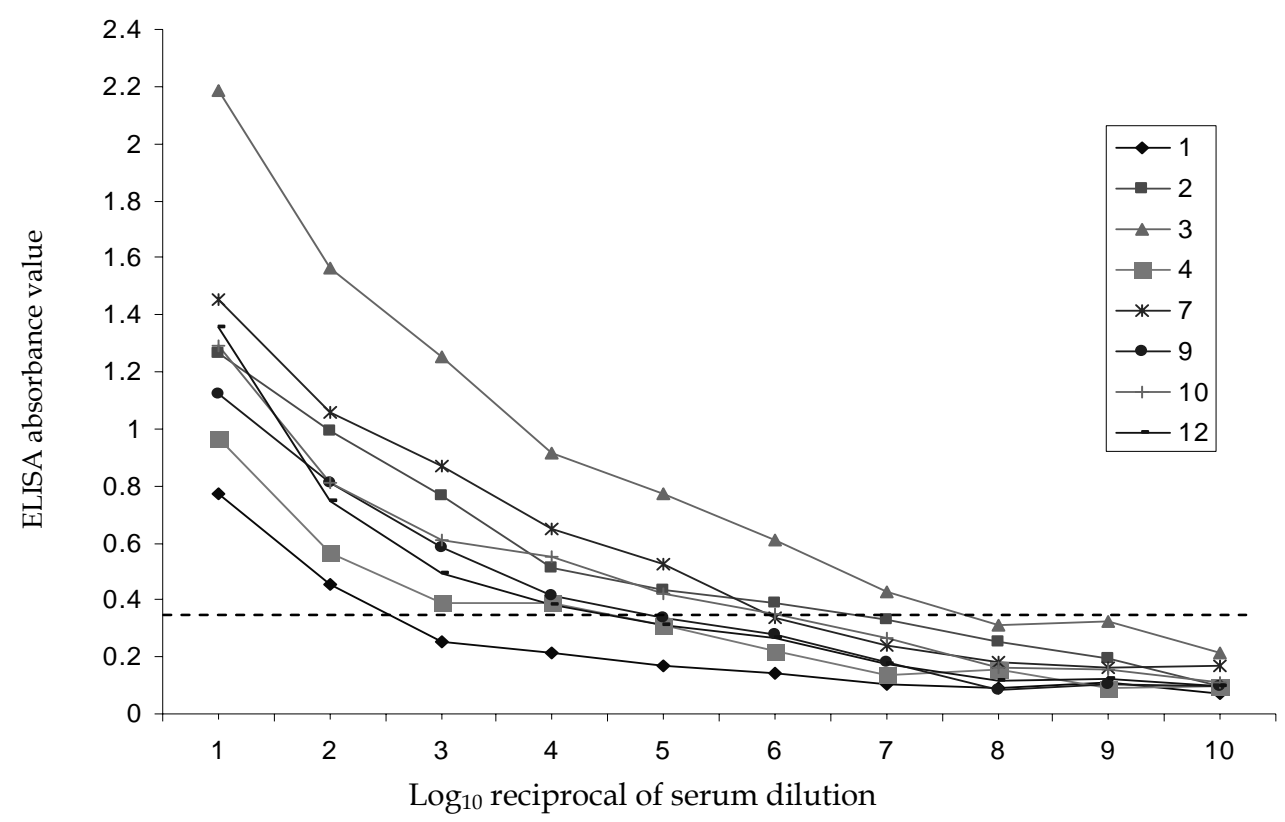

Fig. 1. Standard serial dilution method of determining predicted NDV antibody ELISA titres from corrected absorbance with a positive-negative threshold (PNT) baseline. The broken line represents the PNT baseline (drawn at 0.35), and solid line represents eight different positive serum samples with varying titers.

Correlation regression analysis was performed using the absorbance values of eight sera in the single dilutions $(1: 1000)$ and the end-point titre. The results are shown in Fig. 2. A good positive correlation was observed $(\mathrm{r}=0.912, \mathrm{n}=8, \mathrm{P}<0.01)$. The slope was 4.69 and the $\mathrm{Y}$-intercept was 2.26. So, the standard curve equation for prediction of antibody titre from the absorbance value at $1: 1000$ dilutions was as follows:

$\log _{10}$ titre $=4.69($ Absorbance $1: 1000)+2.26$

To verify the equation, the antibody titre was predicted from the absorbance value at $1: 1000$ dilution of eight selected sera, mentioned above, using the developed formula.

The predicted titres were compared with the corresponding end-point titres (Table 4). Both the titres were very close.

Sensitivity was done by adding different dilution (from $10^{-3}$ to $10^{-10}$ ) of the known diluted hyperimmune serum (dilution at $10^{-3}$ ) on the newly developed In-house Indirect ELISA kit which was coated with purified antigen at a dilution of $10^{-6}$. The serum dilution $10^{-5}$ appeared to be the highest dilution which had the maximum lowest capacity to bind with the coated antigen of the present ELISA kit. This indicated that the antibody in the serum was absent above $10^{-5}$ dilution resulting the serum could not be bound with the coated 
antigen of the ELISA plate. Sensitivity test of the newly developed In-house Indirect ELISA kit also revealed the positivity of different serum samples which were negative in haemagglutination inhibition (HI) test. The newly developed In-house Indirect ELISA kit can measure as minimum as $8 \mathrm{HI}$ unit titre present in the tested serum samples. Williams et al. (1997) were reported that the sensitivity of the developed Indirect ELISA kit was $97.2 \%$.

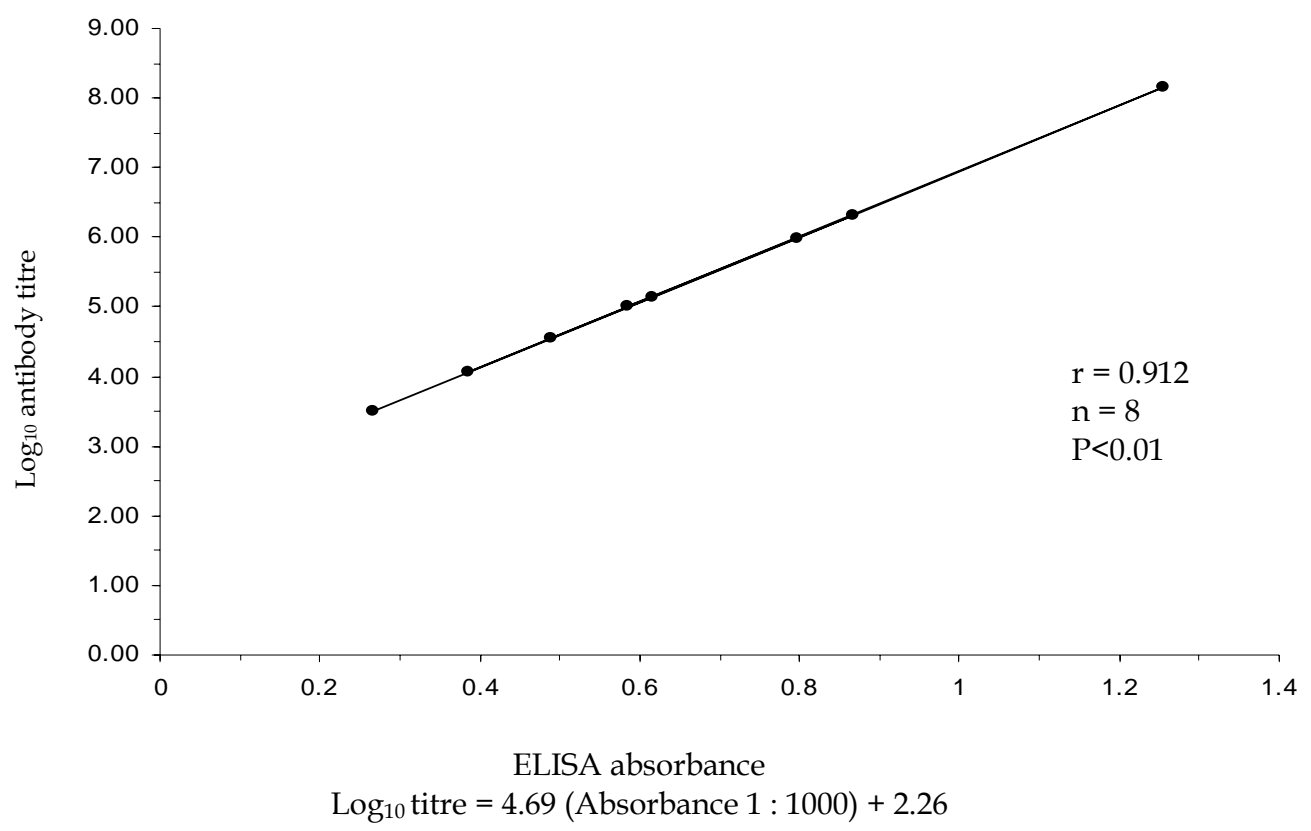

Fig. 2. Relationship between the predicting ELISA antibody titre of eight sera in ELISA and their corrected absorbance at a $1: 1000$ dilution. Correlation coefficient and regression equation are shown

Table 4. NDV antibody titres of eight different sera determined by different methods from absorbance values measured in a single dilution (1:103)

\begin{tabular}{l|c|c|c}
\hline Sample No. & Absorbance values & End-point titre & Predicted antibody titre \\
\hline 1 & 0.266 & 2.5 & 3.51 \\
2 & 0.796 & 6.7 & 5.99 \\
3 & 1.256 & 7.7 & 8.15 \\
4 & 0.385 & 4.5 & 4.07 \\
7 & 0.867 & 5.9 & 6.33 \\
9 & 0.586 & 4.8 & 5.01 \\
10 & 0.615 & 6.1 & 5.14 \\
12 & 0.489 & 4.5 & 4.55 \\
\hline
\end{tabular}


Specificity was performed by adding different known serum like NDV and IBDV at $10^{-3}$ dilution on the newly developed In-house Indirect ELISA kit which was coated with $10^{-6}$ dilution of purified antigen of NDV. The serum only anti-NDV was found to bind with the coated antigen instead of serum of IBDV in the plate which revealed the high specificity of the newly developed In-house Indirect ELISA kit. 97.2\% specificity of the developed Indirect ELISA kit was reported by the Williams et al. (1997).

The performance of the newly developed In-house Indirect ELISA system was compared with that of a commercial ELISA kit (Biochek, NDV antibody detection ELISA kit). A microtitre plate showing color development in Biochek ELISA is shown in Plate 1. A total of 80 chicken sera obtained from the vaccinated and recovered flocks of birds from commercial broiler and layer farms were tested by both the ELISA system as shown in Plate 1 and 2. The titre was predicted for each serum. The correlation between the titres predicted by the two systems were analysed by correlation-regression analysis. The results are shown in Fig. 3. There was a significant positive correlation $(\mathrm{r}=0.901, \mathrm{n}=80, \mathrm{P}<0.01)$ between titres determined by the two methods. The ELISA titres detected by In-house Indirect ELISA were in general slightly higher than that of commercial ELISA kit (Biochek NDV antibody detection kit). This was probably due to relatively higher background reaction in the In-house Indirect ELISA because of the use of crude antigen.

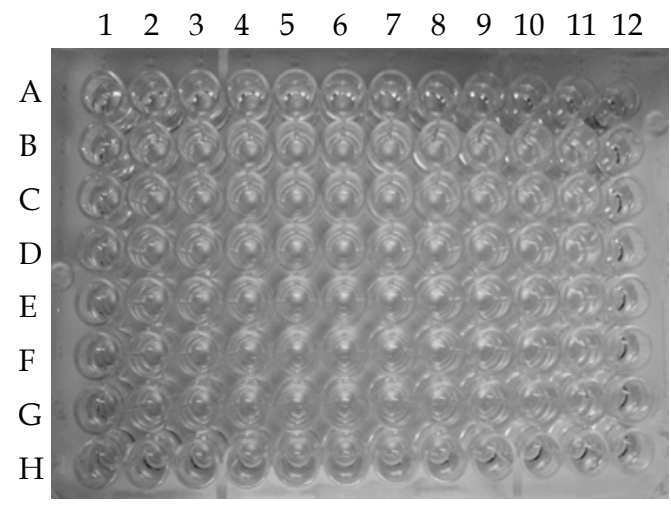

Plate 1. A Biochek NDV antibody detection ELISA plate showing colour development

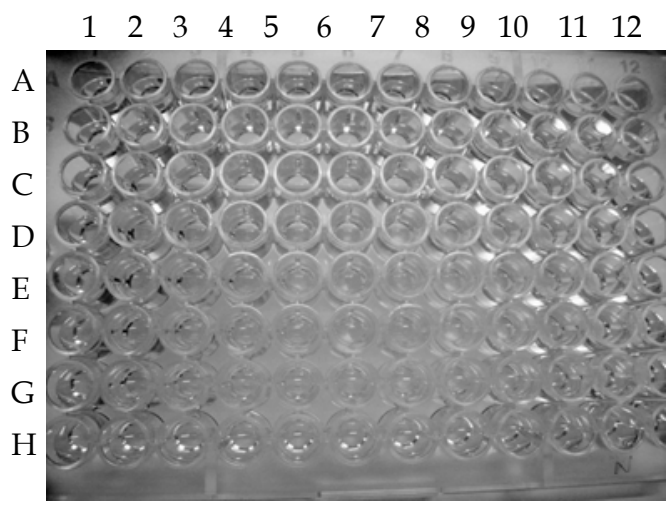

Plate 2. A In-house NDV antibody detection ELISA plate showing colour development

Overall results of the present study indicate that further studies may be required to make the newly developed In-house Indirect ELISA kit more acceptable and popular in the field of determination of antibody titre of serum of chicken vaccinated against NDV to the poultry population of Bangladesh that are to evaluate and improve the sensitivity and specificity of the newly developed In-house Indirect ELISA kit, it is necessary to use various types of serum samples of the layer and broiler chicken of different farm and the computational method should be further optimized using larger number of sera samples using two-fold instead of ten-fold dilutions of serum. 


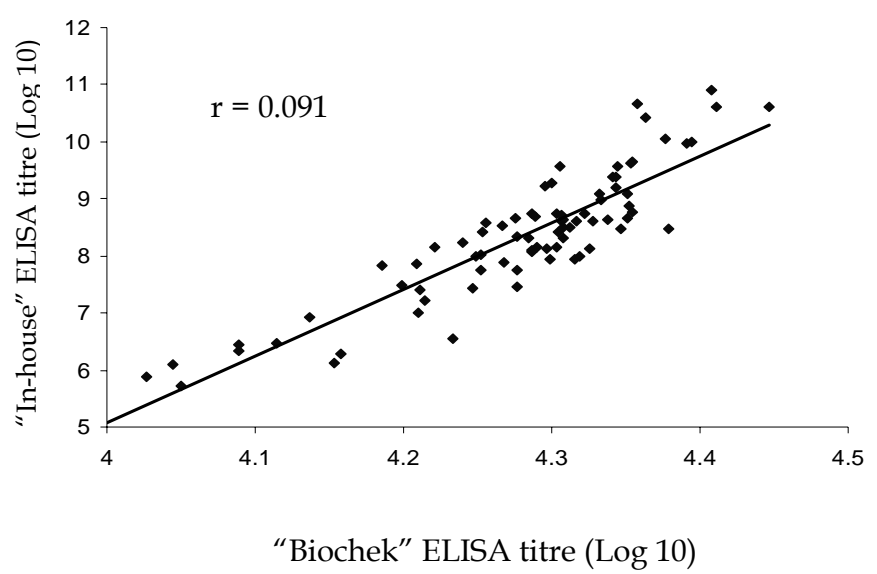

Fig. 3. Correlation between the titres determined by "In-house" and "Biochek” ELISA methods

The present study also had some limitations such as the eggs; chickens used for this study were not taken from the SPF flocks, which might have played some role in getting the exact result in the detection and serological studies. While the goal of development of Inhouse Indirect ELISA kit is to achieve a simpler, more reliable, sensitive, specific, economic, rapid serological test that is combined with maximum operator safety.

\section{REFERENCES}

Alexander, D. J. 1991. Newcastle disease and other paramyxovirus infections, in diseases of poultry. $9^{\text {th }}$ edn, edited by B. W. Calnek, H. J. Barnes, C. W. Beard, W. M. Reid and H. W. Jorder Jr. Ames, Iowa, USA: Iowa State University Press, pp. 496-519.

Alexander, D. J. 1997. Newcastle disease and other avian paramyxoviridae infections, in diseases of poultry. $10^{\text {th }}$ edn, edited by B. W. Calnek, H. J. Barnes, C. W. Beard, L. R. McDougald \& Y. M. Saif. Ames, Iowa, USA : Iowa State University Press, pp. 541-569.

Brown, J., Resurreccion, R. S. and Dickson, T. G. 1990. The relationship between the hemagglutination-inhibition test and the enzyme-linked immunosorbent assay for the detection of antibody to Newcastle disease. Avian Dis., 34: 585-587.

Folitse, R., Halvorson, D. A. and Sivanandan, V. 1998. A dot immunoblotting assay (Dot Blot ELISA) for early detection of Newcastle disease antibodies in chickens. Avian Dis., 42: 14-19.

Snyder, D. B., Marquardt, W. W., Mallinson, E. T. and Russek, E. 1982. Rapid serological profiling by enzyme-linked immunosorbent assay. I. Measurement of antibody activity against Newcastle disease virus in a single dilution. Avian Dis., 27(1): 161-170.

Tizzard, L. 1982. An introduction to veterinary immunology. Sussex. England Saunder Company Published.

Williams, R., Boshoff, C. H., Verwoerd, D., Schoeman, M., Wyk, A. V., Gerdes, T. H. and Roos, K. 1997. Detection of antibodies of Newcastle disease virus in ostriches (Struthio camelus) by an indirect ELISA. Avian Dis., 41: 864-869. 